\title{
Evaluation the effect of royal jelly on the growth of two members of gut microbiota; Bacteroides fragillis and Bacteroides thetaiotaomicron
}

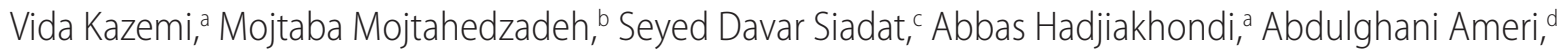 \\ Sara Ahmadi Badi, ${ }^{c}$ Azadeh Manayi, ${ }^{a^{*}}$ and Mahdi Bagheri ${ }^{\mathrm{b}}$
}

\author{
aMedicinal Plants Research Center, Faculty of Pharmacy, Tehran University of Medical Sciences, Tehran, Iran. \\ bDepartment of Pharmacotherapy, Faculty of Pharmacy, Tehran University of Medical Sciences, Tehran, Iran. \\ cMicrobiology Research Center (MRC), Pasteur Institute of Iran, Tehran, Iran. \\ dDepartment of Food and Drug Control, Ahvaz Jundishapur University of Medical Sciences, Ahvaz, Iran. \\ *Correspondence to Azadeh Manayi (email: manayi@sina.tums.ac.ir). \\ (Submitted: 05 September 2018 - Revised version received: 18 September 2018 - Accepted: 10 October 2018 - Published online: 26 February 2019)
}

\begin{abstract}
Objective In this study the effect of Royal jelly on the growth of two important members of Bacteroides spp.; Bacteroides fragilis and Bacteroides thetaiotaomicron, was evaluated. Also the physicochemical properties and cytotoxicity effects of Royal jelly on Caco-2 cell line as gastrointestinal epithelial cell model, was assessed.

Methods Bacteria, $B$. fragilis and B. thetaiotaomicron were grown on brain heart infusion (BHI) broth medium supplemented with Royal jelly in three different concentrations $(2.5 \%, 5 \%$ and $10 \% \mathrm{v} / \mathrm{v})$, both of the bacteria $\left(1.5 \times 10^{8} \mathrm{cfu} / \mathrm{mL}\right)$ were inoculated to $\mathrm{BHI}$ broth contained Royal jelly in anaerobic condition. To calculate the bacterial optical density (OD), the absorbance was measured at $600 \mathrm{~nm}$ after an overnight. Also Caco-2 cells, was used to study the effects of Royal jelly on epithelial cell viability, and the physicochemical properties consist of total proteins, polysaccharides, phenolic compounds, total lipids, ash and moisture by UV-Vis spectrophotometric and gravimetric methods were evaluated.

Results The growth of B. fragillis and B. thetaiotaomicron were increased by Royal jelly (2.5\%, $5 \%$ and $10 \% \mathrm{v} / \mathrm{v}$ concentrations) and the results indicated that Royal jelly increased the growth of bacteria in a dose dependent manner $(p<0.001)$. In addition MTT assay showed more than 95\% viability of Caco-2 cells treated with Royal jelly. The Iranian Royal jelly sample contains 59.01\% water, 11.57\% proteins, $12 \%$ lipids, $12.63 \%$ polysaccharide, and 5\% mineral.

Conclusion This study showed that Royal jelly has a potential effect in the preserving gut microbiota and it is suggested that Royal jelly as a complementary and alternative medicine can be used to treatment diseases are associated with gut microbiota-host interactions and immune regulating. Although we need to expand our knowledge by designing clinical trials to confirm the therapeutic effects of Royal jelly on gut microbiota modulation as a barrier function.

Keywords bacteroides fragile, bacteroides thetaiotaomicron, gastrointestinal tract microbiota, gut microbiota, royal jelly, traditional medicine
\end{abstract}

\section{Introduction}

Royal jelly (RJ) as a natural product and healing compound has been widely used since ancient times in the treatment of diseases and health promotion in many countries. This substance is a complex mixture of water, proteins, fats, carbohydrates, amino acids, mineral salts, vitamins, enzymes, hormones, trace elements and antibiotics. RJ has been used as a supplement due to its rich carbohydrate, protein, and mineral ingredients. ${ }^{1-3}$ According to the recent pharmacological studies, RJ has antioxidant, antitumor, anti-inflammatory, antiallergic, antiaging, and antihypertensive activities. It has been indicated that oral ingestion of RJ ameliorated lipoprotein metabolism and decreased serum total cholesterol and low-density lipoprotein levels. This product may play an important role in the maintenance of the gastrointestinal function due to its components and antioxidant activity. The bacteria are the major part of the adult gut microbiota with an essential metabolic function to degrade polysaccharides of plants. Further, formation of the intestinal mucosal barrier and stimulation of angiogenesis within a gut are other beneficial properties of the bacteria for human. ${ }^{1-3}$ The microbial community of the digestive tract is known as microbiota which is a combination of various micro-organisms and thousands of bacterial species. ${ }^{4,5}$

Gut microbiota are involved in maintaining the gastrointestinal tract function. They also modulate the synthesis of vitamins, metabolism of substances, inhibition of pathogenic bacteria growth, stimulation of mucosal IgA production, modulation of immune system, and maintenance of human hemostasis. ${ }^{6-9}$ Bacteroides fragillis and Bacteroides thetaiotaomicron belong to the phylum of Bacteroidetes. Bacteroides spp. are abundance in gut microbiota and have significant roles in complex community. Bacteroides spp. mostly colonizes in the distal of GI where fermentation of indigestible carbohydrates occurs. ${ }^{10,11}$

Bacteroides spp. have significant role on the host metabolism and immunity through degradation of proteins and carbohydrate complex and activating the regulatory $\mathrm{T}$ cells. Furthermore, gut microbiota especially Bacteroides spp. have an important role in regulation of immune system and maintenance of homeostasis. ${ }^{5,11,12}$ The patterns in the growth of bacterial in the gastrointestinal tract can be affected by various diseases, drugs, and diet. Recent studies have indicated that the effect of enteral nutrition on the reduction of infectious morbidity in critically ill patients was significantly higher than parenteral nutrition. ${ }^{14}$ Therefore, the modification of gut microbiota-host interactions by Royal jelly (viable benefited bacteria) is controversial.

However, it is suggested that RJ could modulate gut microbiota, the balance between species of living bacteria in human gastrointestinal tract and effect on homeostasis and host functions. 
In this study the physicochemical properties of Iranian Royal jelly and its effect on the growth of $B$. fragillis and $B$. thetaiotaomicron, as important parts of gastrointestinal microbiota and also the cytotoxicity effect of Royal jelly on Caco-2 cell line as gastrointestinal epithelial cell model evaluated.

\section{Materials and Methods}

\section{Evaluation the Physiochemical Properties of Royal Jelly}

\section{Royal jelly preparation}

Freshly harvested Royal jelly applied from beekeepers of Damavand reign, Tehran province $\left(35.7013^{\circ} \mathrm{N}, 52.0586^{\circ} \mathrm{E}\right)$ was used.

\section{Royal jelly composition}

The composition of $\mathrm{RJ}$ is depended on seasonal and regional conditions; $100 \mathrm{mg}$ of RJ sample were put on small amount of water and to dissolve using ultrasonic homogenizer, subsequently the volume reached to $100 \mathrm{~mL}$ with deionized water in a volumetric flask to obtain concentration of $1 \mathrm{mg} / \mathrm{mL}$. Total phenol was measured using UV-Vis spectrometer (Optizen, Korea) according to the method of Folin-Ciocalteau. ${ }^{15}$ The total polysaccharide was analyzed using UV-Vis spectrometer (Optizen, Korea) according to the previous study conducted by Vazirian et al. ${ }^{16}$ Bradford method was applied to determine total protein content of the sample spectrophotometrically. ${ }^{17}$ Total lipid was measured based on the described method by Müller et al. (2000). ${ }^{18}$ Moisture and ash content of the sample was measured according to the previous study conducted by Horwitz and Latimer (2000). ${ }^{19}$

\section{Evaluation the Cytotoxicity Effect of Royal Jelly}

\section{Cell culture}

The human epithelial cell line IBRC C10094 Caco-2 was purchased from Iranian Biological Resource Center. The cells were grown in Dulbecco's modified eagle medium (high glucose; $\mathrm{Gibco}^{\mathrm{TM}}$, USA), supplemented with $10 \%$ fetal bovine serum (Gibco $\left.{ }^{\mathrm{TM}}, \mathrm{USA}\right), 1 \%$ penicillin/streptomycin $\left(\mathrm{Gibco}^{\mathrm{TM}}\right.$, USA) and incubated at $37^{\circ} \mathrm{C}$ in a $5 \% \mathrm{CO}_{2}$ atmosphere. Cells were routinely subcultured every 3 or 4 days by trypsinization until they reached approximately $80 \%$ confluence. $^{20}$

\section{MTT assay}

Caco- 2 cells were cultured at the density of $2 \times 10^{4}$ cell/well in a 96-well culture plate and incubated overnight, before RJ treatment. Cells were treated with RJ and incubated for $24 \mathrm{~h}$. Then, the cell culture medium was changed. Cells were incubated with $100 \mu \mathrm{L}$ MTT contained medium for $4 \mathrm{~h}$, the medium was removed and then $100 \mu \mathrm{L}$ DMSO was added. The absorbance was measured at $550 \mathrm{~nm}$, using a microplate spectrophotometer (Epoch ${ }^{\mathrm{TM}}$, USA). ${ }^{20}$

\section{Evaluation the Effect of Royal Jelly on Bacterial Growth}

Bacterial strains and growth condition

Bacteroides fragillis ATCC 23745 and B. thetaiotaomicron ATCC 10774 were grown on blood agar plates containing brain heart infusion (BHI) broth supplemented with hemin $(5 \mu \mathrm{g} / \mathrm{mL})$ and menadione $(1 \mu \mathrm{g} / \mathrm{mL})$. Incubation performed at $37^{\circ} \mathrm{C}$ under anaerobic conditions provided $80 \% \mathrm{~N}_{2}, 10 \%$ $\mathrm{CO}^{2}$, and $10 \% \mathrm{H}_{2}$ atmosphere. ${ }^{21}$
Treatment bacteria with medium contained Royal jelly

Samples of RJ solution were prepared by deionized distilled water. Sterilization of RJ solution was performed using a $0.22-\mu \mathrm{m}$ pore size polyvinylidene difluoride filter (Millipore, Billerica, MA, USA). This sterile solution was aseptically added to BHI broth at different concentrations (2.5\%, $5 \%$ and $10 \% \mathrm{v} / \mathrm{v})$. After preparation of $1.5 \times 10^{8} \mathrm{cfu} / \mathrm{mL}$ bacterial suspension, $100 \mu \mathrm{L}$ of $B$. fragillis and $B$. thetaiotaomicron suspension were inoculated to BHI broth and RJ contained BHI broth. Incubation was performed overnight in anaerobic condition. Finally, the bacterial optical density was calculated by measuring the absorbance at $600 \mathrm{~nm}$ after an overnight.

\section{Ethics Statement}

This work was part of the research that has been performed in accordance with the ethical rules of the Helsinki Declaration and all the experiments and procedures used in this research were approved by the Ethics Committee of Tehran University of Medical Sciences (No.IR.TUMS. REC.1395.2643).

\section{Statistical Analysis}

Significant differences between groups were determined by one-way ANOVA followed by Tukey's post-hoc test for multiple comparisons. The level of statistical significance was $p<0.05$.

\section{Results}

The obtained data showed that the total amounts of phenol and polysaccharide in Iranian Royal jelly sample were 21.99 $\pm 0.41 \mu \mathrm{g} / \mathrm{mg}$ GAE and $12.63 \%$, respectively. The contents of lipid and protein were $12 \%$ and $11.57 \%$. The moisture of Royal jelly was $59.01 \%$ and the amount of ash was $0.1 \%$ (Table 1). The results demonstrated that the growth of both the bacteria were increased by RJ supplementation (Table 2). The growth of $B$. fragillis and B. thetaiotaomicron exposed to all concentration of RJ increased in comparison with control group. This product induced growth of $B$. fragillis higher than $B$. thetaiotaomicron in all of the tested concentrations. RJ with concentration of $2.5 \%$ versus $5 \%$ of RJ significantly increased the growth of $B$. fragillis (OD; $0.330 \pm 0.045,0.610 \pm 0.098$, respectively) and $B$. thetaiotaomicron $(\mathrm{OD} ; 0.299 \pm 0.060,0.518 \pm 0.100$, respectively). The results also indicated that there was a significant difference between the growth of $B$. fragillis (OD; $1.020 \pm$ $0.360)$ and $B$. thetaiotaomicron (OD; $0.751 \pm 0.250)$ exposed to $\mathrm{RJ}$ at a concentration of $10 \% \mathrm{v} / \mathrm{v}$ in comparison with the growth of them exposed to RJ at concentration of $5 \% \mathrm{v} / \mathrm{v}$ $(p<0.001,<0.01$, respectively)

The MTT assay indicated that RJ had no cytotoxicity effect on Caco- 2 cells after $24 \mathrm{~h}$. The cell growth inhibition values for RJ against Caco-2 cells were $5 \%$ after $24 \mathrm{~h}$. Result of this study showed that this substance did not reduce cell viability.

\begin{tabular}{|c|c|c|c|c|c|}
\hline Phenol & Polysaccharide & Protein & Lipid & Moisture & Ash \\
\hline $21.99 \pm 0.41^{\mathrm{a}}$ & $12.63 \pm 0^{b}$ & $11.57 \pm 0^{b}$ & $2.00 \pm($ & $59.01 \pm 0^{b}$ & $.1 \pm 0^{b}$ \\
\hline
\end{tabular}

aicrogram per milligram Gallic acid equivalent. bercentage (\%). 
Table 2. The effect of different concentrations (OD) of Royal jelly on the growth of B. fragillis and B. thetaiotaomicron versus control (non-exposed bacteria to RJ)

\begin{tabular}{|c|c|c|c|c|}
\hline \multirow{2}{*}{ Bacterial strain } & \multirow{2}{*}{ Control } & \multicolumn{3}{|c|}{ Royal jelly concentration (\%v/v) } \\
\hline & & 2.5 & 5 & 10 \\
\hline B. fragillis & $0.102 \pm 0.020$ & $0.330 \pm 0.045^{*}$ & $0.610 \pm 0.098^{* * *+\dagger}$ & $1.020 \pm 0.360^{* * *,+t+, 895}$ \\
\hline B. thetaiotaomicron & $0.116 \pm 0.020$ & $0.299 \pm 0.060^{*}$ & $0.518 \pm 0.100^{* * * *+1+}$ & $0.751 \pm 0.250^{* * *,+t, \$ \S}$ \\
\hline
\end{tabular}

"Significant difference between the control versus others.

tSignificant difference between bacteria exposed to RJ 2.5\% versus bacteria exposed to other concentrations.

${ }^{5}$ Significant difference between bacteria exposed to RJ 5\% versus bacteria exposed to RJ $10 \%$.

\section{Discussion}

The present findings indicated that RJ could increase the bacterial growth with no cytotoxicity effect on Caco-2 cells. Intestinal epithelial cells are the interface between gut microbiota and host interactions. Therefore, the effect of Royal jelly on Caco-2 cell viability (a human intestinal epithelial cell model) was evaluated. According to our findings, Iranian RJ contains $59 \%$ water, $11.57 \%$ proteins, $12 \%$ lipids, and up to $12.63 \%$ polysaccharide. Similar to our study, Johansson (1995) indicated that the moisture of RJ was near to $60 \%$. It was also indicated that RJ contained $12 \%$ proteins, $7.6 \%$ lipids, and up to $18 \%$ polysaccharide..$^{11}$ Compared with Johansson's (1995) study, the amount of protein and polysaccharide in our study was low. While, higher lipid contents in the RJ samples was observed in this study.

Another study indicated that RJ from different ecosystems in South America to Europe has the wider standard ranges of components $(60-70 \%$ water, $8-18 \%$ proteins, 9-18\% total polysaccharide, $3-11 \%$ lipids and $0.3-0.5 \%$ ash). The findings of this study also were comparable with those examined samples of different origins. The amount of ash in the sample of our study was lower $(0.1 \%)$ than those reported previously. ${ }^{12,13}$ The amount of phenolic compounds in our sample was near to the findings of Nagai and Inoue $(21.2 \mu \mathrm{g} / \mathrm{mg})$ and Nabas et al. $(23.3 \mu \mathrm{g} / \mathrm{mg}))^{11,13}$ Several factors including the plants species, health of the plant, season, and environmental factors can affect the amount of phenolic compounds in RJ. ${ }^{14}$

The main biological and pharmacological activities (antioxidant, immunomodulation and anti-inflammatory effects) of natural antioxidants may be related to their phenolic constituents. It has been suggested that consuming foods rich in phenolic compounds can increase the population of Bacteroides in the gastrointestinal tract (GIT). Therefore, Iranian RJ could be effective for modulation of several functional organs such as gastrointestinal tract. The GIT contains many important beneficial microbes. For example, Bacteroides spp. are one of the micro-organisms present primarily for the sustenance of a healthy GI system. ${ }^{19}$ The biological activity and development of this bacterium is further enhanced in the presence of phenolic compounds. ${ }^{19}$ Some in vitro and in vivo experimental studies on polyphenolic compounds have reported that these compounds hastened the growth of Lactobacillus and Bacteroides and catalyze their probiotic potency in the GIT. ${ }^{20}$ Under in vitro conditions, polyphenolic compounds promoted the increase in the numbers of Bacteroides by 10-100-folds, which was beneficial for the intestinal microbiota. Increasing in Bacteroides leads to a decrease in the formation of ammonia, skatoles and harmful amine procarcinogens in the large intestine, and reduce acid production that raises fecal $\mathrm{pH} .{ }^{21}$ Since microbiota growth was relatively unaffected by most of the aromatic compounds tested, probiotic colonization in the intestine should continue in the presence of phenolic compounds so as to improve the intestinal microbial balance and inhibit pathogen growth. Previous research has demonstrated that consumption of other plants rich in polyphenolic compounds such as green tea selectively promotes the growth of Bacteroides in the gut wall. ${ }^{18}$ This data demonstrated that RJ contains a high percent of phenolic compounds. However, phenolic compounds increase the growth of intestinal bacteria; antimicrobial activities of polyphenols have been demonstrated. ${ }^{21}$ The level of inhibition varies depending on the bacterial species, the chemical structure of the compound and phenolic concentration. Findings of this study showed that RJ could change the bacterial growth. This effect may be related to the RJ concentrations and the bacterial species. It has been known that, gastrointestinal microbiota composition has significant roles in the determination of health and disease states. ${ }^{13}$ As well as scrutiny gut microbiota pattern in conduct an investigation and preclinical studies can be helped in understanding the processes of maintaining gut microbiota by supplementation and prescription of some natural products such as Royal jelly in preserving gut microbiota, especially in ill patients undergoing intensive care and receiving medication and variety of drugs with a microbiota weakening effect. This study was designed based on research to recognizing ways to reinstate gut microbiota composition and results were showed that RJ has a potential effect in the preserving and energy conversation of gut microbiota to improve human health as a defense barrier, and also Royal jelly can be used to formulating preparations for treatment diseases and maintaining health situation. Although this assumption needs to expand our knowledge about the mechanism of effects caused by Royal jelly on the common microbial community includes pathogens and friendly micro-organisms of healthy voluntaries and patients, by designing clinical trials to confirm the preliminary obtained results on the therapeutic effects of Royal jelly on gut microbiota modulation as a barrier function.

\section{Conclusion}

According to the importance of Bacteroides spp. in gut microbiota-host interactions, it is suggested that gastrointestinal microbiota pattern could be changed by Royal jelly supplementation in patients receiving variety of drugs with a gut microbiota weakening side effects. Finding of this work indicated that RJ has a potential effect in the growth of gut microbiota 
which could improve human health and treatment diseases. However, several clinical trials should be done to cover a large number and wide scope of subjects and confirm the beneficial effects of RJ on gut microbiota. In conclusion, Royal jelly seems to be a very effective approach to treat and, all results of low sample size and short-term studies have to be taken into account. In this purpose, multi-center study to evaluate the comprehensive application of Royal jelly with established treatment modalities in a randomized, controlled trial has been started.

\section{Acknowledgments}

This research was supported by the Medicinal Plants Research Center, Tehran University of Medical Sciences and the authors would like to thank Microbiology Research Center (MRC), Pasteur Institute of Iran.

\section{Conflict of Interest}

The authors declare that there is no conflict of interest.

\section{References}

1. Alvarez-Suarez JM, Tulipani S, Romandini S, Bertoli E, Battino M. Contribution of honey in nutrition and human health: a review. Mediterranean J Nutr Metab. 2010;3:15-23.

2. Pasupuleti VR, Sammugam L, Ramesh N, Gan SH. Honey, propolis, and royal jelly: a comprehensive review of their biological actions and health benefits. Oxid Med Cell Longev. 2017;2017:1259510.

3. Rao PV, Krishnan KT, Salleh N, Gan SH. Biological and therapeutic effects of honey produced by honey bees and stingless bees: a comparative review. Rev Bras Farmacogn. 2016;26:657-664.

4. Benson AK, Kelly SA, Legge R, Ma F, Low SJ, Kim J, et al. Individuality in gut microbiota composition is a complex polygenic trait shaped by multiple environmental and host genetic factors. Proc Natl Acad Sci. 2010;107:18933-18938.

5. Clemente JC, Ursell LK, Parfrey LW, Knight R. The impact of the gut microbiota on human health: an integrative view. Cell. 2012;148: 1258-1270.

6. Lozupone CA, Stombaugh Jl, Gordon Jl, Jansson JK, Knight R. Diversity, stability and resilience of the human gut microbiota. Nature. 2012;489: 220-230.

7. Sekirov I, Russell SL, Antunes CM, Finlay BB. Gut microbiota in health and disease. Physiol Rev. 2010;90:859-904.

8. Nicholson JK, Holmes E, Kinross J, Burcelin R, Gibson G, Jia W, et al. Host-gut microbiota metabolic interactions. Science. 2012;336:1262-1267.

9. Prakash S, Tomaro-Duchesneau C, Saha S, Cantor A. The gut microbiota and human health with an emphasis on the use of microencapsulated bacterial cells. J Biomed Biotechnol. 2011;2011:981214.

10. den Besten G, van Eunen K, Groen AK, Venema K, Reijngoud DJ, Bakker BM. The role of short-chain fatty acids in the interplay between diet, gut microbiota, and host energy metabolism. J Lipid Res. 2013;54:2325-2340.

11. Tremaroli V, Bäckhed F. Functional interactions between the gut microbiota and host metabolism. Nature. 2012;489:242-249.

12. Hooper LV, Littman DR, Macpherson AJ. Interactions between the microbiota and the immune system. Science. 2012;336:1268-1273.

13. Lin L, Zhang J. Role of intestinal microbiota and metabolites on gut homeostasis and human diseases. BMC Immunol. 2017;18:2.

14. Ahmadi Badi S, Siadat SD, Khatami SH, Irani SH. Induction effect of bacteroides fragilis derived outer membrane vesicles on toll like receptors gene expression and cytokine concentrations in human intestinal epithelial cell. Cell J. 2019;21:57-61.

15. Elhenawy W, Debelyy MO, Feldman MF. Preferential packing of acidic glycosidases and proteases into Bacteroides outer membrane vesicles. MBio. 2014;5:e00909-e00914.

16. Ahmed WM, Khalaf AA, Moselhy WA, Safwat GM. Royal jelly attenuates azathioprine induced toxicity in rats. Environ Toxicol Pharmacol. 2014;37:431-437.

17. Özan F, Çörekçi B, Toptaş O, Halicioğlu K, Irgin C, Yilmaz F, et al. Effect of Royal Jelly on new bone formation in rapid maxillary expansion in rats. Med Oral Patol Oral Cir Bucal. 2015;20:e651-e656.

18. Metwally Ibrahim SEL, Kosba AA. Royal jelly supplementation reduces skeletal muscle lipotoxicity and insulin resistance in aged obese rats. Pathophysiology. 2018;25:307-315.

19. Boger MCL, Lammerts van Bueren A, Dijkhuizen L.Cross-feeding amongst probiotic bacterial strains on prebiotic inulin involving the extracellular exo-inulinase of Lactobacillus paracasei strain W20. Appl Environ Microbiol. 2018;84:e01539-18.

20. Melok AL, Lee LH, Mohamed Yussof SA, Chu T. Green tea polyphenol epigallocatechin-3-gallate-stearate inhibits the growth of Streptococcus mutans: a promising new approach in caries prevention. Dent J (Basel). 2018;6:E38.

21. Mayta-Apaza AC, Pottgen E, De Bodt J, Papp N, Marasini D, Howard L, et al. Impact of tart cherries polyphenols on the human gut microbiota and phenolic metabolites in vitro and in vivo. J Nutr Biochem. 2018;59:160-172. 